\title{
Um estudo da automação para redução de perdas na rede de distribuição de água'
}

\section{A study of automation for loss reduction in water distribution system}

\author{
Fritz, Rodrigo Tenório'; Gimenes, Julia2; Pina Filho, Armando Carlos ${ }^{3}$ \\ 1 Universidade Federal do Rio de Janeiro, Programa de Engenharia Urbana, \\ Escola Politécnica, Av. Athos da Silveira Ramos, 149, Cidade Universitária, \\ 21941-485, Rio de Janeiro - RJ, Brasil, rtfritz@poli.ufrj.br \\ 2 Universidade Federal do Rio de Janeiro, Programa de Engenharia Urbana, \\ juliagimenes@poli.ufrj.br \\ 3 Universidade Federal do Rio de Janeiro, Programa de Engenharia Urbana, \\ armando@poli.ufrj.br
}

\begin{abstract}
RESUMO
Este trabalho apresenta um estudo das possibilidades de aplicação da automação no sistema de abastecimento de água urbano, com intuito de reduzir perdas no sistema, em diferentes pontos, bem como aumentar o controle da concessionária sobre o mesmo. A gestão do controle e redução de perdas visa contribuir na eficiência do sistema de abastecimento, principalmente na rede de distribuição. A partir da literatura relacionada, são descritos diferentes tipos de perdas de água no sistema e em que ponto ocorre, sendo correlacionados aos seus agentes causadores. São apresentadas soluções para o aumento da eficiência na redução de perdas, principalmente no controle da pressão e vazão no sistema, que é apontado como fator diretamente relacionado às perdas. A setorização e a automação, através de dispositivos conectados remotamente à uma central de controle, são apresentadas como soluções ótimas para maior controle e rapidez na identificação de falhas. Os casos encontrados sugerem que a automação gera grandes vantagens para o prestador, que aumentaria a oferta de água de seu sistema produtor, além da redução de custos pela grande redução de perdas e aumento da eficiência do sistema.
\end{abstract}

Palavras-chave: abastecimento de água, automação, redução de perdas.

\section{ABSTRACT}

This work presents a study of the possibilities of application of automation in urban water supply system, in order to reduce losses in the system, at different points, as well as increase the control of the concessionaire. The management of the control and loss reduction aims to contribute in the efficiency of the supply system, mainly in the distribution system. From the literature, are described different types of water losses in the system and at what point will occur, being

\footnotetext{
${ }^{1}$ FRITZ, Rodrigo Tenório; GIMENES, Julia; PINA FILHO, Armando Carlos. Um estudo da automação para redução de perdas na rede de distribuição de água. In: II SIMPÓSIO NACIONAL DE GESTÃO E ENGENHARIA URBANA: SINGEURB, 2019, São Paulo. Anais... Porto Alegre: ANTAC, 2019.
} 
correlated to their causative agents. Solutions are presented for increased efficiency in reducing losses, especially in the control of the pressure and flow rate in the system, which is touted as a factor directly related to losses. The sectoring and automation, via devices connected remotely to a central control, are presented as great solutions for greater control and speed identification of faults. The cases found suggest that the automation generates great advantages to the service provider, which would increase the supply of water from the system manufacturer, besides the reduction of costs by the great reduction of losses and increase of the efficiency of the system.

Keywords: water supply, automation, water-loss reduction.

\section{INTRODUÇÃO}

Globalmente, a demanda de água tem crescido e a oferta, diminuído. Perdas de água em redes de abastecimento, sempre presentes nas operações hidráulicas, são uma das características no gerenciamento operacional, mesmo em sistemas com infraestrutura bem desenvolvida e de boas práticas operacionais. Entretanto, nos países em desenvolvimento, a combinação de fraca infraestrutura, esgotamento sanitário ineficaz e abastecimento intermitente pode significar um alto risco para saúde da população. Perdas de água nas redes de abastecimento são um problema mundial, que requer um gerenciamento estratégico e que pode ser universalmente aplicado. O problema na oferta de água acaba por impulsionar as ações no controle de perdas em determinados países e cidades. A Inglaterra e o Japão são referências mundiais.

Fonseca (2011) destaca a atuação dos ingleses que detêm conhecimento adquirido através de aplicações no próprio Reino Unido e prestação de consultoria em vários países, assim como na autoria de livros. Todos os prestadores de água do Reino Unido exercem uma prática ativa no gerenciamento e controle de perdas, porém são as indústrias/companhias de água da Inglaterra e do País de Gales que foram os pioneiros em mecanismos e tecnologia no ganho de um melhor entendimento do controle de perdas.

No Japão, especialmente nas zonas urbanas onde se observa a escassez de água, a importância de planejar o uso eficiente e racional dos recursos vem sendo amplamente reconhecida pela população. Em 1964, a cidade de Tóquio enfrentou uma seca sem precedentes. Desde então o Japão começou a se preocupar com o aproveitamento eficiente e racional da água. Os órgãos e companhias regionais de abastecimento de água se mobilizaram para promover ações para reduzir desperdícios de água, causados principalmente por vazamentos (PENA, 2010).

No Brasil, segundo dados do Sistema Nacional de Informações sobre o Saneamento (SNIS), para a média nacional dos índices de perdas na distribuição (INO49), que incluem perdas reais e aparentes, têm-se níveis próximos de $40 \%$ nos últimos oito anos (PERTEL, 2014). Sendo assim, é importante buscar soluções para reduzir as perdas no sistema de abastecimento de água, e o presente trabalho visa realizar um estudo das possibilidades de aplicação da automação para mitigar o problema. Esse estudo foi feito a partir de uma revisão bibliográfica sobre os temas relacionados: sistema de abastecimento de água, controle de perdas e aplicação da automação. Como resultado, espera-se comprovar que a automação fornece ferramentas poderosas que auxiliam o administrador a gerenciar a rede de distribuição, disponibilizando mais água com qualidade e menos perdas.

\section{SISTEMA DE ABASTECIMENTO}

Um sistema convencional de abastecimento de água é constituído das seguintes unidades: captação, estação de tratamento, reservação e redes de distribuição. Sistemas de abastecimento de água podem ser vistos como sistemas produtivos que transformam água bruta (matéria prima) em água tratada (produto final), fornecida ao consumidor por meio de um serviço tarifado. Essas etapas são ilustradas na Figura 1. 
Figura 1 - Ełapas de um Sistema de Abastecimento de água.

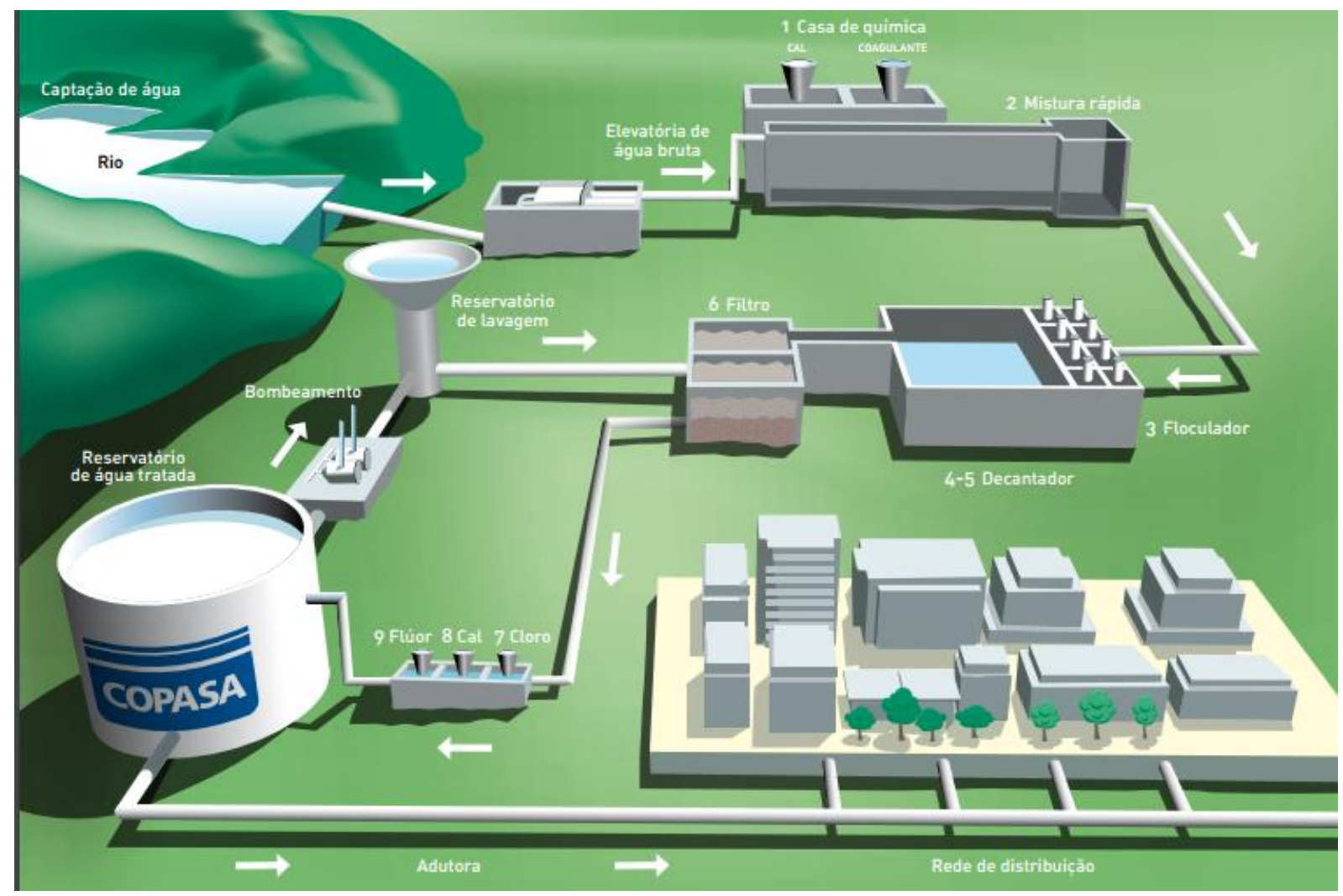

Fonte: COMUSA (2018)

A captação de água é realizada em rios, lagos e represas, ou subterrânea, através de poços artesianos. A água captada é enviada para a estação de tratamento, seja por gravidade ou bombeamento, para receber os devidos procedimentos buscando tornar a água própria para o consumo. Após o tratamento da água nas ETAs, a água é armazenada em reservatórios, para então ser distribuída. As redes de distribuição representam a ramificação que a água passa até chegar às residências e indústrias.

Para que a rede funcione corretamente, é necessário que a água seja pressurizada. Quando a pressão na rede é baixa, instalam-se bombas para elevar essa pressão e levar a água em todos os locais da rede. Em locais com elevada pressão, as válvulas reguladoras são de suma importância, para evitar que ocorram danos à tubulação e perdas por vazamento.

\subsection{Perdas de Água no Sistema}

A International Water Association (IWA) define perdas de água como: volume de água correspondente à diferença entre a água que entra no sistema de abastecimento e o consumo autorizado. As perdas de água podem ser calculadas para todo o sistema ou para subsistemas, seja na fase de água não tratada (da captação ao tratamento) até as zonas de distribuição. As perdas de água dividem-se em perdas reais e perdas aparentes.

As perdas estão relacionadas aos vazamentos. Na maioria dos sistemas bem operados, somente $3 \%$ são vazamentos visíveis e $97 \%$ são não visíveis. A ocorrência de vazamentos em tubulações de diâmetros maiores que $150 \mathrm{~mm}$ são mais visiveis e de fácil detecção. Já na ocorrência de pequenos vazamentos em tubulações de menor porte e, principalmente, localizadas próximas a córregos, riachos ou galerias de águas pluviais, tem-se uma situação crítica, pois o vazamento não se apresenta mais tão visível ou de fácil localização, podendo 
permanecer ali por meses ou anos, até a sua detecção e conserto (TROJAN e KOVALESKI, 2005).

\section{CONTROLE DE PERDAS NO SISTEMA}

O controle de pressão é fundamental para a redução de perdas reais em um sistema de abastecimento de água, pois a pressão é um dos principais fatores que influenciam o número de vazamentos e a vazão dos mesmos. É necessário minimizar as pressões do sistema e a faixa de duração de grandes pressões e assegurar padrões necessários ao atendimento dos consumidores. A Figura 2 apresenta a síntese das características dos vazamentos e as ações para seu controle.

Figura 2 - Síntese das ações para o controle e a redução de perdas reais.

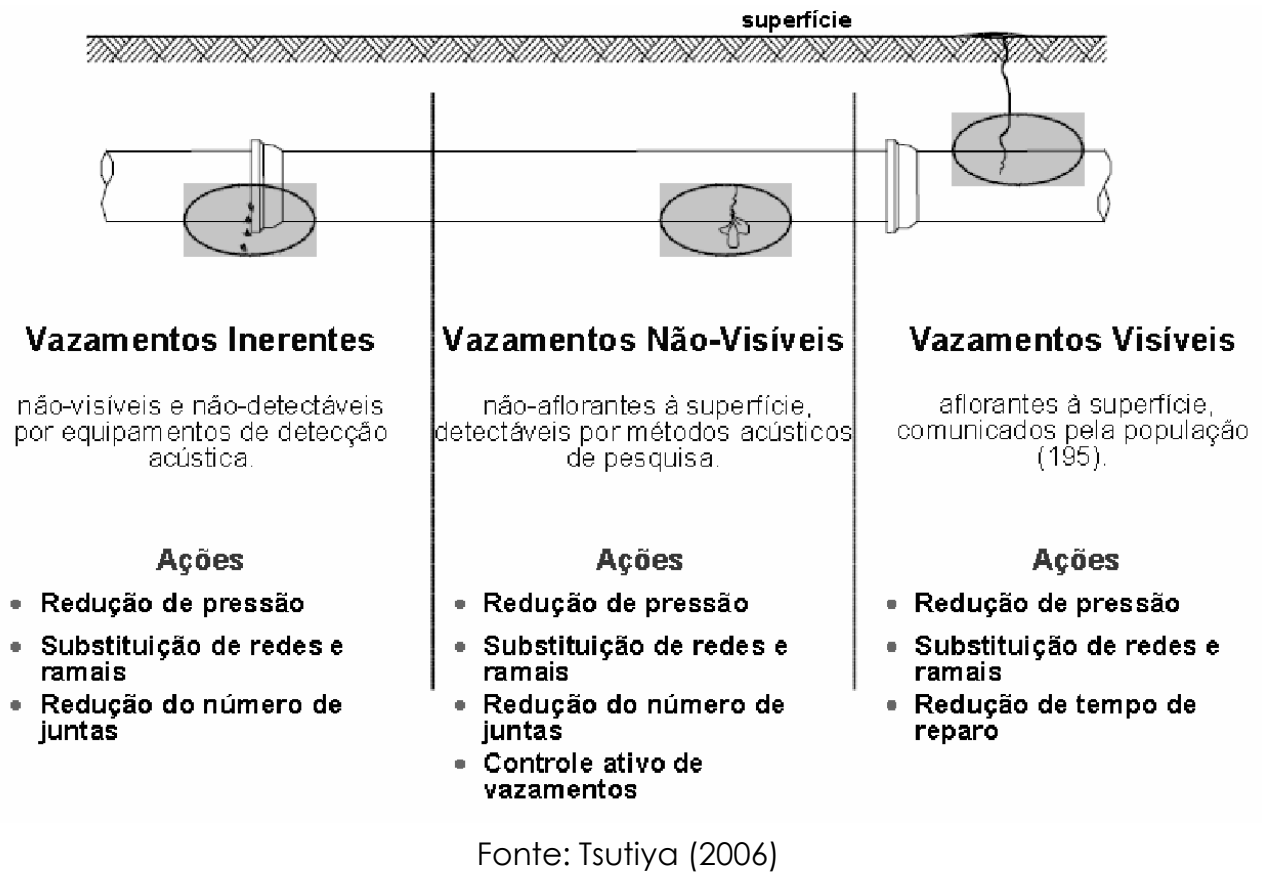

Segundo Pena (2010), a utilização de sistemas de controle de pressão através de softwares possibilita diminuição significativa de perdas reais. Além de altas pressões, outro problema é a oscilação da pressão na rede de distribuição. Esses eventos podem causar fraturas, rupturas e fadiga em tubulações, expulsão de juntas de vedação, flexão e movimentação de tubos. A adoção de um programa de controle de pressões na rede de distribuição pode reduzir os arrebentamentos de tubulações.

Para um melhor controle de pressões nas redes de distribuição é importante que o sistema seja setorizado por áreas. A setorização consiste na separação do sistema de abastecimento hídrico urbano em distritos denominados zonas pitométricas, com o objetivo geral de estabelecer um controle mais efetivo das pressões e das vazões de água destas zonas (FONSECA, 2011).

Quanto aos vazamentos, o controle ativo (Active Leakage Control) é o modo operacional no qual o prestador implanta recursos e equipamentos para detectar de forma ativa vazamentos não visíveis, que não foram relatados por consumidores ou outros meios.

Ações de combate às perdas aparentes podem ser visualizadas na Figura 3, a saber: macromedição, gestão comercial e micromedição.

\subsection{Automação na Distribuição}

O conceito de automação em sistemas de abastecimento de água assemelha-se muito ao que acontece no setor elétrico. Da mesma forma que esse segmento pode ser dividido em 
geração, transmissão e distribuição de energia, o setor de saneamento envolve o tratamento de água, o transporte para os reservatórios e a distribuição aos consumidores.

Figura 3 - Ações de controle para perdas aparentes.

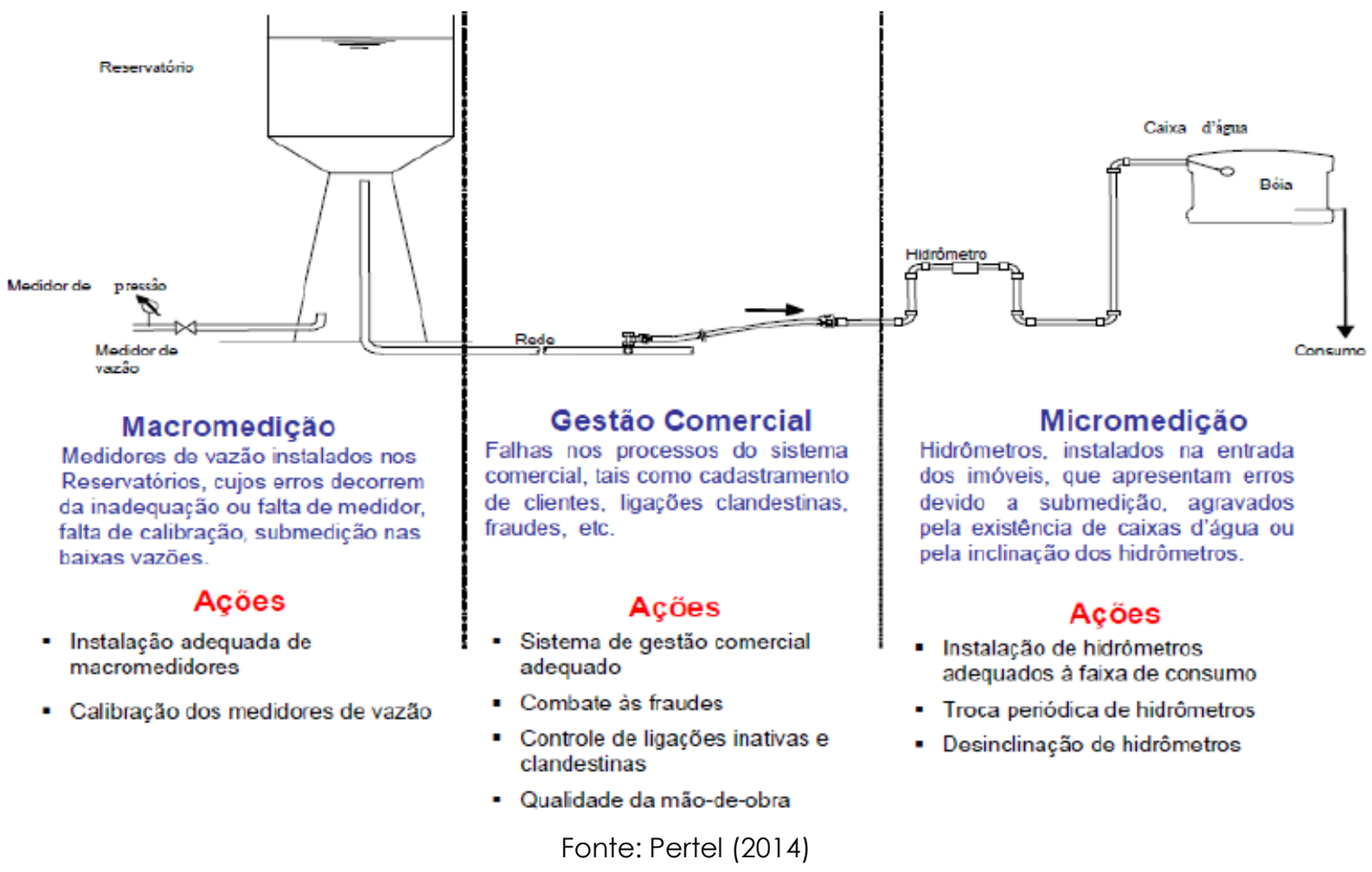

A instalação de válvulas redutoras de pressão (VRPs) possibilita que a pressão seja estabilizada, o que diminui a fadiga das tubulações e proporciona uma redução do número de vazamentos, contribuindo para redução das perdas reais do sistema de abastecimento. As VRPs podem estar conectadas ao sistema de controle e automação, bem como os sensores de vazão nas tubulações.

Mário Filho (2001) ressalta que a automação em saneamento soluciona uma série de dificuldades operacionais. Por meio da telemetria e controladores lógicos programáveis (CLPS), várias unidades podem ser assistidas a partir de um centro de controle operacional (CCO) com funções de operação, otimização e planejamento, com menor custo e maior segurança. Além disso, é possível verificar uma melhoria do sistema com a automação, através do monitoramento por sensores, como visto em Allen et al. (2013) e Silva Júnior (2017).

A automação pode representar também um ganho ambiental significativo na conservação dos recursos naturais, auxiliando na manutenção e preservação de águas limpas, além da preservação dos mananciais. Dessa forma, projetos que visem uma melhor qualidade da distribuição, evitando perdas e furtos, além de uma otimização do sistema, proporcionam qualidade de vida para uma região.

Alguns dos resultados alcançados com o uso da automação, segundo Trojan e Kovaleski (2005), são a redução da pressão média nas tubulações transportadoras, a rapidez e qualidade nos reparos dessas tubulações, a formação de uma base de informações para a criação de programas para novas instalações e para melhorias no sistema, citados na literatura como essenciais para o controle do índice de perdas e a qualificação do trabalho.

Apesar de todas essas vantagens, a automação em saneamento ainda é pontual e isso é reflexo da falta de recursos das companhias de saneamento, majoritariamente estatais. Todavia, já existem casos bem sucedidos, em âmbito nacional, na aplicação da automação para redução de perdas, realizados nos últimos 10 anos. Podem ser citados, no começo da década, os estudos de Vinciguera (2009) e Oliveira et al. (2009), em Campo Grande (MS) e 
Poços de Caldas (MG), respectivamente, e mais recentemente, os casos de São Francisco (BA) e Região dos Lagos (RJ), apresentados pela AESBE (2018) e FAPESP (2018). Os custos para implantação dos sistemas automatizados foram variados, dependendo do tamanho da rede, e a redução de perdas ficou em torno de 2 a $5 \%$, o que pode parecer pouco, mas no caso da Região dos Lagos (RJ), por exemplo, o percentual equivale a 2,5 milhões de metros cúbicos de água por ano.

\section{CONSIDERAÇÕES FINAIS}

A grande vantagem na utilização da automação no sistema é a redução das perdas de água e, consequentemente, a preservação dos mananciais e da oferta de água, além da redução de custos, pois reduz diretamente a quantidade de água a ser tratada, o que reduz também o consumo de produtos químicos e energia necessários para o tratamento.

Com a implantação de sistemas automatizados, criando microzonas de controle, é possível direcionar as ações de forma assertiva, otimizando as ações de combate às perdas, reduzindo os recursos hídricos necessários para o abastecimento da região, além de uma maior eficiência na utilização de produtos químicos, redução da necessidade de ampliação do sistema e, consequentemente, o impacto ao meio ambiente. Além disso, reduz a mão de obra em campo destinada à verificação de pressão e eventuais anomalias, o que diminui também o custo de operação e torna o sistema mais eficiente e enxuto.

Também há redução da possibilidade de furtos e vazamentos no sistema, pois o controle fica mais robusto e preciso. A automação traz benefícios de amplo monitoramento, pois com os sensores é possível acompanhar com maior velocidade as variáveis do sistema.

Concluindo, a automação fornece ferramentas poderosas que auxiliam o administrador a gerenciar a rede de distribuição. Com isso se disponibiliza mais água com qualidade e menos perdas, o que pôde ser comprovado por estudos de casos realizados na última década.

\section{REFERÊNCIAS}

AESBE. Associação Brasileira das Empresas Estaduais de Saneamento. Automação do sistema adutor do São Francisco (BA), da Embasa, reduz custos e perdas de água. Disponível em: <http://www.aesbe.org.br/automacao-do-sistema-adutor-do-sao-francisco-ba-da-embasareduz-custos-e-perdas-de-agua>. Acesso em: 05 abr. 2018.

ALLEN, M.; PREIS, A.; IQBAL, M.; WHITTLE, A. J. Water distribution system monitoring and decision support using a wireless sensor network. In: 14th IEEE/ACIS International Conference on Software Engineering, Artificial Intelligence, Networking and Parallel/Distributed Computing, Hawaii, Estados Unidos, julho de 2013, p. 641-646.

COMUSA. Serviços de Água e Esgoto de Novo Hamburgo. Tratamento de Água. Disponível em: <http://www.comusa.rs.gov.br/index.php/saneamento/tratamentoagua>. Acesso em: 18 ago. 2018.

FAPESP. Fundação de Amparo à Pesquisa do Estado de São Paulo. Software possibilita controlar pressão de água em rede de distribuição. Disponível em:

<http://pesquisaparainovacao.fapesp.br/software_possibilita_controlar_pressao_de_agua_e m_rede_de_distribuicao/542>. Acesso em: 30 jan. 2018.

FILHO, M. Automação no saneamento básico: diferentes necessidades para um mesmo objetivo. Revista Controle \& Instrumentação, ed. 61, São Paulo: Valete, jul. 2001.

FONSECA, F. R. Modelo de sistema de automação aplicado à setorização de redes de abastecimento hídrico. Tese de doutorado. Universidade de São Paulo, São Paulo, 2011. 
IWA. International Water Association. International Best Practice Water Balance and Performance Indicators for Water Supply Services. Disponível em: <https://iwa-network.org>. Acesso em: 18 ago. 2018.

OLIVEIRA, F. G. R.; REIS, F. A. G. V.; GIORDANO, L. C.; MEDEIROS, G. A. Controle de perdas em sistema de abastecimento de água: o Caso do Município de Poços de Caldas (MG).

Engenharia Ambiental - Espírito Santo do Pinhal, v.6, n.1, p.309-320, jan./abr. 2009.

PENA, M. M. Aplicação e análise da metodologia da IWA para o controle de perdas no sistema de abastecimento de água da baixada de Jacarepaguá/RJ. Tese de doutorado. Universidade Federal do Rio de Janeiro, COPPE, Rio de Janeiro, 2010.

PERTEL, M. Experimentos Hidráulicos Conjugados ao Uso de Indicadores de Desempenho Aplicados à Quantificação de Perdas em Sistemas de Abastecimento de Água no Brasil. Tese de doutorado. Universidade Federal do Rio de Janeiro, COPPE, Rio de Janeiro, 2014.

SILVA JÚNIOR, J. F. Detecção de perdas em sistemas de distribuição de água através de rede de sensores sem fio. Dissertação de Mestrado. Universidade Federal de Pernambuco, Programa de Pós-Graduação em Engenharia Elétrica, 2017. 166 p.

TROJAN, F.; KOVALESKI, J. L. Automação no abastecimento de água: Uma ferramenta para redução de perdas e melhoria nas condições de trabalho. In: XII Simpósio de Engenharia de Produção (SIMPEP), Bauru, São Paulo, novembro de 2005.

TSUTIYA, M. T. Abastecimento de Água. 4a ed. São Paulo: Departamento de Engenharia Hidráulica da Escola Politécnica da Universidade de São Paulo, 2006. 643 p.

VINCIGUERA, V. Avaliação do Impacto da Redução de Pressão nas Perdas Reais em Setor de Distribuição de Água do Município de Campo Grande - MS. Dissertação de Mestrado. Universidade Federal de Mato Grosso do Sul, 2009. 81 p. 